\title{
Kernos
}

Revue internationale et pluridisciplinaire de religion grecque antique

4 | 1991

Varia

\section{A. Iriarte, Las redes del enigma. Voces femininas en el pensamiento griego}

\section{Emilio Suárez de la Torre}

\section{OpenEdition \\ Journals}

Édition électronique

URL : http://journals.openedition.org/kernos/328

DOI : $10.4000 /$ kernos.328

ISSN : 2034-7871

\section{Éditeur}

Centre international d'étude de la religion grecque antique

\section{Édition imprimée}

Date de publication : 1 janvier 1991

Pagination : 340-343

ISSN : 0776-3824

\section{Référence électronique}

Emilio Suárez de la Torre, «A. Iriarte, Las redes del enigma. Voces femininas en el pensamiento griego », Kernos [En ligne], 4 | 1991, mis en ligne le 11 mars 2011, consulté le 24 septembre 2020. URL : http://journals.openedition.org/kernos/328 ; DOI : https://doi.org/10.4000/kernos.328 
si cela allait de soi, qu'il faut considérer comme authentiques toutes les propositions d'Euclide, même (surtout ?) celles qui sont généralement tenues pour apocryphes.

Beaucoup plus gênante, me semble-t-il, est la certitude, nombre de fois formulée, de l'existence d'une filiation spirituelle directe entre la pensée "mathématico-religieuse» des prêtres égyptiens et la philosophie grecque. C'est ainsi que Platon ne fait qu'unir sa voix aux légendes et aux mythes immortalisés par les prêtres d'Amon dans les temples, et que les Dialogues de Platon, la Métaphysique d'Aristote et les Éléments d'Euclide traduisent en grec cet ensemble de mythes et de légendes.

L'image que l'A. se fait de la civilisation égyptienne est aussi pour le moins curieuse, donnant souvent l'impression de se rattacher aux courants théosophique et symboliste. Ainsi les prêtres égyptiens auraient délibérément caché sous une forme cryptographique, dans une volonté ésotérique, leurs plus belles pensées, position qu'il est impossible de soutenir, même pour les textes ptolémaïques. De même, l'aspect énigmatique de certains monuments ou légendes de l'Égypte devient plus clair, selon l'A., si l'on aborde ceux-ci sous l'angle mathématique. Particulièrement révélateur est l'interprétation symbolique que l'A. donne d'un récit aussi connu que la Bataille de Qadech, où il prend pour argent comptant tout ce que le texte contient de formulaire, sans connaître l'apport pourtant substantiel de la critique moderne. Cela l'amène, entre autres, à voir dans le corps de l'armée d'Amon un principe mathématique dianoétique!

En résumé, par sa méthode éclectique et ses préjugés philosophiques, cet ouvrage n'intéressera peut-être pas tant les spécialistes de l'antiquité ou des mathématiques que les historiens de ces deux disciplines.

Jean WINAND (Liège)

Ana IRIARTE, Las redes del enigma. Voces femininas en el pensamiente griego, Madrid, Taurus Humanidades, 1990, 161 p., 11 images, avec une Préface de Nicole Loraux.

La Préface de Nicole Loraux (Directrice de la thèse de troisième cycle de l'EHESS de Paris qui est le fondement du livre) est sans doute la meilleure présentation de ce livre, qualifié par elle de "stimulant" et "terriblement convaincant".

Dans son introduction (p. 17-35), l'A. fait une révision sommaire des données historiques qui témoignent de la condition marginale de la femme grecque, sans oublier quelques remarques à propos des hétaïres. Par contre, les documents purement littéraires, en commençant par Hésiode, nous offrent un portrait particulier de la femme, avec un lien très étroit entre les activités féminines (p. ex. tisser), sa métis caractéristique et son image extérieure. Mais c'est surtout le théâtre tragique 
qui va nous dessiner le portrait le plus complet de la condition du "féminin" pour les Athéniens : les femmes tragiques "prennent la parole» apparemment contre ce qui est propre à leur statut social, mais c'est une parole voilée, énigmatique. La voix féminine aura aussi son espace "naturel" grec dans l'expression oraculaire, domaine traditionnel du langage énigmatique. C'est ainsi que la voix des femmes, à qui est interdit l'espace ouvert de la polis, trouvera une troisième voie entre le silence et le discours.

Le chapitre I (Los dioses indican, p. 37-73) développe plusieurs des idées précédentes dans le parcours diachronique de l'évolution de la mantique grecque. Parfaitement en harmonie avec le développement de cette mantique du point de vue de la tradition grecque, l'A. fait remarquer comment les différents changements qui se sont produits dans la manière divine de se manifester sont en rapport avec des aspects centraux de sa thèse : de la Terre prophétisante à Zeus avaleur de la métis et marié à Thémis, nous assistons à l'apparition d'une conception du pouvoir prophétique associé à la souveraineté. Il faut remarquer l'intérêt de l'analyse que l'A. fait des passages de l'épopée où la souveraineté de Zeus se montre toujours en rapport avec des fonctions prophétiques. L'analyse sémantique des familles de $\varphi \rho \alpha ́ \zeta \omega$ et de $\sigma \eta \mu \alpha i v \omega$, avec leurs nombreuses nuances, d'un côté, et l'étude des "paires complémentaires" Agamemnon/Nestor, Achille/Patrocle et Hector/Polydamas d'un autre, constituent des points vraiment brillants dans l'ensemble du chapitre. L'A. souligne aussi comment la mantique apollinienne est associée à la "parole énigmatique" comme moyen de la communication entre les dieux et les hommes.

Les dernières pages de ce chapitre proposent des réflexions sur les Dialogues Pythiques de Plutarque, surtout en ce qui concerne la forme des réponses delphiques et les rapports entre énigme et poésie, ce qui sert à démontrer que la responsabilité de l'obscurité des réponses doit être cherchée (du point de vue des penseurs Grecs) du côté de la Pythie.

Les Voces femeninas del oráculo est le titre du chapitre II (p. 75-114), qui contient d'abord une étude des opinions reflétées par les Dialogues Pythiques de Plutarque. L'A. déduit de ces opinions "qu'il y a deux manières pour le dieu d'intervenir : par la Pythie-poétesse, associée à la Muse, et par la porte-parole du dieu 'pédagogue'» (p. 82). Une autre conclusion, concernant les problèmes des oracles en vers et du pneuma est que le modèle établi par Plutarque «relègue le pneuma, et le vers, à la période initiale du sanctuaire»; ces procédés-ci seront adoptés par la mantique apollinienne qui les abandonnera ensuite au bénéfice de la "parole signifiante», du style prosaïque, issu d'une communication directe entre le dieu et la Pythie, sans l'intervention du pneuma (voir nos objections ci-dessous). L'A. pense que Plutarque reflète "l'histoire d'une expropriation, à savoir celle de la Terre par Apollon; l'oracle 
"verbal" du dieu l'aurait donc emporté sur les «images nocturnes" et autres moyens de communication de la Déesse Mère. En d'autres termes, l'A. voit ici la défaite du principe féminin (même, ajoute-t-elle, si celui-ci s'est manifesté).

À mon avis, cette partie du chapitre contient des arguments un peu faibles, surtout en ce qui concerne la forme des réponses. La raison en est une certaine confusion dans les niveaux chronologiques établis. Il est clair que le vers et le pneuma ne sont pas des traits "pré-apolliniens" adoptés par le culte delphique et puis abandonnés. La mantique inspirée est justement la mantique apollinienne. Celle-ci, ainsi que la figure de la Pythie «sibylline» inspirée et la relation du vers "cryptique» avec les réponses oraculaires, ont probablement surgi en Grèce simultanément et avant le VIIle siècle av. J.-C. C'est une question tout à fait différente de celle de la succession Gé-Apollon (d'ailleurs pas absolument éclaircie), à laquelle il ne faut pas faire appel pour arriver à la conclusion de l'expropriation. Modestement je n'irais pas au-delà d'une séquence mantique technique-mantique inspirée (je renvoie aux études de B.C. Dietrich, par exemple, Oracles and Divine Inspiration, in Kernos, 3 [1990], p. 157-174).

Ce même chapitre nous présente des analyses beaucoup plus attirantes du personnage des Pythies de la tragédie (en l'occurrence surtout les Euménides d'Eschyle et l'Ion d'Euripide), où, du point de vue du recenseur, le lecteur trouvera les remarques les plus pertinentes et les mieux fondées de ce livre. Une conclusion importante concerne la fonction de la Pythie chez ces auteurs en tant que "celle qui 'présente' aux consultants les images employées par le dieu pour transmettre sa pensée" (p. 104). Une belle analyse de la figure de Cassandre complète la présentation de ces femmes-prophétesses et amène l'A. à regarder virginité et ignorance comme les traits principaux que leur attribuent les penseurs Grecs, avec une dépendance très grande du dieu à l'égard des devins masculins, plus "sages».

Le chapitre III (Entre le silencion y el discurso, p. 115-130) tente de démontrer, encore une fois par des exemples extraits surtout de la tragédie, que pour les Grecs la $\sigma \alpha \varphi \eta j v \varepsilon i \alpha$ appartient au discours des hommes (dont l'attribut exclusif est le logos ouvert face à la cité), tandis que l'ambiguité et, par conséquent, le langage énigmatique, est le trait caractéristique des femmes, en rapport très étroit avec l'univers et les activités des femmes dans l'oikos (mais le langage prophétique - et énigmatique - de l'Orestie n'est pas l'apanage exclusif des femmes : cf. la parodos de l'Agamemnon chantée par des vieillards). Très intéressantes sont les remarques sur le rapport parole énigmatiquevêtements de noces-suicide dans les Suppliantes d'Eschyle et d'Euripide. 
Le dernier chapitre (La Esfinde rapta y profetiza, p. 131-144) constitue un bel exemple d'étude, à l'appui de l'archéologie, de la signification de la sphinge si inquiétante pour les Grecs (dont les représentations ont un caractère "sexuel" très bien mis en évidence par l'A.). L'analyse des témoignages littéraires amène l'A. à conclure à l'évolution du monstre ravisseur à la chanteuse d'énigmes : une ö $\lambda$ vo dont l'énigme est conçue à l'opposé des chants poétiques qui donnent la gloire, et qui, par sa nature, est très proche de la parole prophétique. En ce sens Edipe, élevé hors de la ville et différent des autres jeunes de sa communauté, sera le rival ultime qui déliera la trame des mots tressés par elle, dans une victoire qui, tout en le faisant apparaître comme

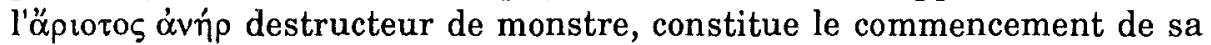
propre ruine.

Dans ses Conclusions (p. 145-148), l'A. souligne les points qui confirment le rôle assigné à la parole des femmes comme intermédiaire entre le silence et le discours des hommes, celui-ci étant doué de logos: en somme, une parole énigmatique qui, dit-elle, appartenait à la tyrannie (mais cette idée devrait être plus fermement soutenue).

Le thème pourrait se prêter à des excès de nature diverse. Néanmoins, l'A. fait une belle démonstration de modération et d'intelligence. Loin de l'énigme inquiétante, nous voici devant un logos bien soutenu...

Il faut se féliciter de cette contribution espagnole à la connaissance de la conception de la femme dans l'Antiquité.

Emilio SUÁREZ DE LA TORRE (Valladolid)

Robert LAFFINEUR (éd.), Thanatos. Les coutumes funéraires en Égée à l'âge du Bronze. Actes du Colloque de Liège (21-23 avril 1986), Liège, Université, 1987, 245 p., 59 pl. (Aegaeum. Annales d'archéologie égéenne de l'Université de Liège, 1).

The study of death, of burial customs and post-funerary practices, has a twofold potential. It may advance understanding both of beliefs about after-life and of the attitudes and structures, social, religious, technological, of the world of the living. Burial evidence can thus be interrogated, empirically and theoretically, for each of these objectives. In sum the two enquiries become unitary, since in the pre-modern world Jenseitsvorstellungen influenced substantially the form and practice of day-to-day living : consider how much of economic production and energy expenditure was for the world of the dead, not the living.

In prehistoric Aegean studies the empirical investigation of death does not have a bad record. Cemeteries of Souphli and Zarkos, Athens, Dendra, Eileusis, Mycenae, Perati and Prosymna, the Cyclades, Kos, Rhodes and Knossos have been published in considerable detail and 